\title{
संगीत शिक्षण में श्रव्य-दृश्य साधनों का उपयोग
}

\section{रुबी विज}

रिसर्च फैलो, गुरु ग्रंथ साहिब अध्ययन विभाग, गुरु नानक देव विश्वविद्यालय, अमृतसर

प्राचीन काल में शिक्षा एक गुरु के द्वारा शिष्य को मौखिक रूप से दी जाती थी। शिष्य गुरु के ध्यान से सुनता रहता था तथा कभी-कभी यह प्रक्रिया नीरस हो जाती थी। वर्तमान शिक्षा शास्त्रियों का मानना है कि विद्यार्थी निष्क्रिय रहकर विधिवत् ढंग से ज्ञान नहीं प्राप्त कर सकता। साथ-साथ हर शिक्षक अपने अध्यापन को सुधारने के लिए प्रयत्न करता है तथा उसके द्वारा वह अच्छा शिक्षक बनने की भी इच्छा रखता है।

इन उद्देश्यों की पूर्ति के लिए जहाँ एक ओर विद्यार्थियों की ज्ञानेन्द्रियों को सक्रिय करते हैं, वहीं दूसरी तरफ अपने अध्यापन को सुधारने के लिए सहायक सामग्री का शिक्षण में उपयोग किया जाता है। इस सहायक सामग्री से एक लाभ यह होता है कि इससे शिक्षण रूचिकर, सरस, आकर्षक एवं उद्देश्य पूर्ण हो जाता है। आधुनिक शिक्षण में सहायक सामग्री हर विषय में प्रयोग की जाती है। संगीत शिक्षण में भी सहायक सामग्री का प्रयोग करना उतना ही आवश्यक हो गया है जितना की अन्य विषयों में, क्योंकि बाकी सभी विषयों से संगीत अधिक रोचक, मनोरंजन आकर्षक एवं लोकप्रिय है।

संगीत का ज्ञान इतना विस्तृत है कि इसकी प्राप्ति पूर्ण रूप से प्रत्यक्ष अनुभव द्वारा नहीं हो सकती। इसमें प्रतिरूपों की सहायता लेनी पड़ती है, जो दृश्य-श्रव्य साधनों से प्राप्त होती है। दृश्य और श्रव्य जो ज्ञानेन्द्रियों में सम्बन्धित होने से इन्हें श्रव्य-दृश्य साधन कहा जाता है। मनुष्य दो इन्द्रियों द्वारा सीखता है:- कर्ण एवं नेत्र। जो काणों द्वारा सुना जाए से श्रव्य तथा जो नेत्रों द्वारा देखा जाए उसे दृश्य साधन कहा जाता है। "Teaching aid is any material aid that affects our sense organs and helps in making learning meaning-ful and interesting. A teaching aid can be any material aid that affects any of our sense organs whereas an audiovisual aid affects the auditory and visual sense organs."

संगीत के क्षेत्र में दृश्य-श्रव्य साधनों का प्रयोग संगीतमय वातावरण उत्पन्न कर शिक्षण को सरल रोचक बनाने के लिए किया जाता है। संगीत के वाद्य जैसे तानपुरा, हरमोनियम, तबला इत्यादि को सहायक सामग्री समझा जाता है। ये सांगीतिक विषय है। अगर संगीत शिक्षण में इन वाद्यों की जानकारी देनी हो तो, इनके एक-एक हिस्से के बारे में समझाने के लिए विद्यार्थियों को प्रत्यक्ष 
दिखाते हुए इसे सहायक सामग्री का ही रूप दिया जाएगा। संगीत में प्रयोग होने वाले दृश्य-श्रव्य साधनों को मुख्यत् तीन वर्गो में बांटा जा सकता है।

दृश्य सामग्री- दृश्य सामग्री का तात्पर्य उन साधनों से है जिनमें केवल दृश्य इन्द्रियों का प्रयोग होता है अर्थात् जिनमें ज्ञान मुख्यतः दृश्य इन्द्रियों के द्वारा प्राप्त होता है। दृश्य साधन इस प्रकार है:

1. वास्तविक पदार्थः वास्तविक पदार्थों का तात्पर्य मूल वस्तुओं से है। वास्तविक पदार्थ विद्यार्थियों की इन्द्रियों को प्रेरणा देते हैं तथा इन्हें निरीक्षण एवं परीक्षण के अवसर प्रदान करके उनकी अवलोकन ;त्रेमतअंजपवदद्ध शक्ति का विकास करते हैं जैसे संगीत शिक्षक तन्त्री वाद्यों को करवाते हुए वाद्यों को विद्यार्थियों के समझ रख कर शिक्षण दे सकता है। वीणा का पाठ करवाते हुए विद्यार्थियों के समक्ष वीणा रखकर समझाए जिससे विद्यार्थी अन्य वाद्यों से वीणा के अन्तर को समझ सकें। अगर वीणा सामने होगी तो विद्यार्थी उसे ज्यादा अच्छी तरह से समझ सकेंगे।

2. नमूने (Model): नमूने वास्तविक पदार्थों अथवा मूल वस्तुओं के छोटे रूप होते हैं। इनका प्रयोग उस समय किया जाता है जब वास्तविक पदार्थ या तो उपलब्ध न हो। उदाहरणतय कई बार शिक्षक अधिक वाद्य नहीं खरीद सकता क्योंकि वाद्य बहुत मंहगे होते है या कई वाद्य बिल्कुल लुप्त हो चुके हैं तो अध्यापक उनके नमूने बनाकर विद्यार्थियों के समक्ष रख सकता है। इस दृष्टि से नमूनों का सबसे बड़ा लाभ यह है कि इनकी सहायता से विद्यार्थियों को ऐतिहासिक, भौगोलिक तथा वैज्ञानिक सभी प्रकार के तथ्यों का ज्ञान सरलतापूर्वक दिया जा सकता है। इनका प्रयोग करते समय अध्यापक को विद्यार्थियों के समझ वास्तविक पदार्थों के आकार का बोध करा देना चाहिए। इससे उनकी वास्तविक पदार्थों के प्रति गलत धारणाएं नहीं बनेंगी।

3. चित्र: चित्रों का प्रयोग उस समय किया जाता है जब न तो वास्तविक पदार्थ ही उपलब्ध हों और न ही नमूने मिल सकें। दूसरे शब्दों में, जब वास्तविक पदार्थों तथा नमूनों का मिलना कठिन हो जाता है तो इस र्थिति में चित्रों का मिलना कठिन हो जाता है तो इस स्थिति में चित्रों का प्रयोग किया जाता है। संगीत शास्त्र पढ़ाने के लिए चित्र बहुत लाभकारी सिद्ध होते हैं। "संगीत में कलाकरों के सम्बन्ध में चर्चा करने के लिए चित्रों का प्रदर्शन अनिवार्य हो जाता है, अन्यथा छात्र उस कलाकार की सही कल्पना नहीं कर सकते ${ }^{\prime \prime i i ~ च ि त ् र ~ क ई ~ प ् र क ा र ~ क े ~ ह ो त े ~ ह ै ं ~ ज ै स े ~ फ ो ट ो च ि त ् र, ~ र े ख ा च ि त ् र ~ आ द ि । ~ स ह ी ~}$ वातावरण में खींचे जाने के कारण फोटोचित्र अधिक विश्वसनीय होते हैं। कलाकरों के गायन या वादन करते समय उनकी विभिन्न मुद्राओं के जो चित्र लिए जाते है उनसे विद्यार्थी कलाकारों की तन्मयता तथा उनकी भावनाओं के अभिव्यक्तिकरण के ढंग का सहज अनुमान लगाते है। 
4. मानचित्र: मानचित्र की सहायता से विद्यार्थियों को सम्बन्धित संगीतकार के विशेष स्थान का ज्ञान करवा सकते है। मानचित्र प्रमाणित होने चाहिए और वही अंश दिखाने चाहिए जिनका पाठ्य क्रम से सम्बन्ध हो। यूं जो मानचित्र बने बनाए बाज़ार से मिल जाते हैं, पर शिक्षक मानचित्र स्वयं बनाए तथा सुन्दर ढंग से अंकित करे तो और भी अच्छा रहेगा।

5. ग्राफ: ग्राफ का अपना निजी महत्व होता है, "ग्राफ का प्रदर्शन 1957 में नई दिल्ली में हुए संगीत सैमीनार में संगीत नाटक अकादमी द्वारा हुआ। 1960 में लगभग इसी प्रकार का संगीत सैमीनार मद्रास में संगीत नाटक संगम द्वारा हुआ था। ${ }^{\prime \text { iii }}$

6. चार्ट: चार्टो के प्रयोग से शिक्षक को शिक्षण का उद्देश्य प्राप्त करने में बड़ी सहायता मिलती है। सैद्धान्तिक एवं क्रियात्मक दोनों प्रकारों के पाठों के लिए चार्ट महत्वपूर्ण है। संगीत में चार्ट की उतनी ही उपयोगिता है जितनी की अन्य विषयों में। ष्राग वर्गीकरण, स्वर स्थान, ध्वनि, नाद, जाति, समय, चक्र इत्यादि विषयों को चार्ट के माध्यम से स्पष्ट किया जा सकता है।"iv चार्ट द्वारा प्रदर्शित की हुए विषय वस्तु सुन्दर, सुडौल तथा मोटी होनी चाहिए ताकि कक्षा के प्रत्येक विद्यार्थी का ध्यान उसकी ओर आकर्षित हो जाए तथा शिक्षण अपने प्रयोजन को आसानी से स्पष्ट कर सके।

7. श्यामपट्ट: कक्षा अध्यापन में दृश्य साधन रूप में श्याममट्ट ही सर्वाधिक प्रयोग में आता है। इसका उचित सही एवं विधिपूर्वक उपयोग पाठ को प्रभावशाली बनाने में बहुत सहायक होता है। शिक्षक पाठ की मुख्य बातें व सारांश श्यामपट्ट पर लिख कर विद्यार्थियों का ध्यान आकर्षक कर सकता है। वादन के बोल, स्वर लिपि, मात्राएं इत्यादि सूक्ष्म बातें श्यामपट्ट की सहायता से करवाए जा सकते हैं। विशेषकर स्वर लिपि चिन्हों को सिखाते समय श्यामपट्ट अत्यन्त आवश्यक सामग्री है। किसी ताल की दुगुन, तिगुन, चौगुन करवानी हो तो अध्यापक मात्रा, विभाग तथा लयकारियां लिख कर विद्यार्थियों को समझा सकता है।

8. बुलैटिन-बोर्ड: बुलैटिन-बोर्ड शिक्षा का एक उपयोगी उपकरण है। संगीत कक्ष में इस पर रचनात्मक कार्य प्रदर्शित किया जा सकता है। सांगीतिक चित्रों, संगीत सम्बन्धी पत्रिकाओं व समाचार पत्रों की कतरनों आदि को प्रदर्शित करके विद्यार्थियों की जिज्ञासा को इस प्रकार से उकसाया जाता है कि उनके ज्ञान में निरन्तर वृद्धि होती रहे तथा वे संगीत सीखने के हमेशा तत्पर रहें। इस पर प्रदर्शित की जाने वाली सामग्री की रूचि, मानसिक स्तर तथा आयु एवं योग्यता के अनुसार होनी चाहिए तथा इसकी अवधि अधिक लम्बी नहीं होनी चाहिए।

9. फ्लेनेल बोर्ड: आधुनिक युग में फ्लेनेल बोर्ड का भी विशेष महत्व है। इसकी बनाने में 36 "x48" के एक प्लाईवुड अथवा हार्डबोर्ड के टुकड़े पर इतने ही नाप के एक फ्लेनेल के कपड़े को 
खींचकर बांध दिया जाता है। तत्पश्चात् उस पर चित्रों को खुरदरे कागज़ पर चिपकाकर बोर्ड पर लगाया जाता है। इससे टुकड़े फ्लेनेल बोर्ड पर चिपके रहते हैं और प्रयोग करने के पश्चात् सरलतापूर्वक हटा लिए जाते हैं। संगीत शिक्षण में संगीत अध्यापक विद्यार्थियों द्वारा संगीत की शिक्षण दे सकता है।

10. स्लाइड और फिल्म पट्टियाँ: विभिन्न प्रकार के वाद्यों एवं कलाकरों की जानकारी इन स्लाइड और फिल्म पट्टियाँ द्वारा दी जा सकती है। "नई दिल्ली स्थित राष्ट्रीय शिक्षा अनुसंधान और प्रशिक्षण के दृश्य-श्रव्य शिक्षा विभाग से संबंधित स्लाइडें प्राप्त की जा सकती हैं।"v इन स्लाइडों द्वारा सूक्ष्म से सूक्ष्म बातों को पर्दे पर बड़ा करके दिखाया जा सकता है। स्लाइड हमेशा शीशे की होती हैं। अंतः इसका प्रयोग बहुत सावधानी से करना पड़ता है क्योंकि इनके टूटने का भय रहता है तथा यह भारी भी होती है।

\section{श्रव्य साधन}

श्रव्य सामग्री का तात्पर्य उन साधनों से है जिनमें केवल श्रव्य इन्द्रियों का प्रयोग होता है। अर्थात् जिनका ज्ञान मुख्यतः कान द्वारा प्राप्त होता है। इस सामग्री के अन्तर्गत उन उपकरणों की सहायता ली जाती है जो मुख्य रूप से ध्वनि उत्पादक होते हैं तथा जिनके द्वारा कलाकार के गायन तथा वादन को भविष्य के लिए सुरक्षित रखा जाता है। संगीत का ज्ञान अभ्यास के साथ सुन-सुन कर अधिक होता है। और सुनने के लिए कई यन्त्रों का प्रयोग किया जाता है।

\section{रेडियो}

भारत में प्रसारण परम्परा के आरम्भ से पहले संगीत का प्रदर्शन केवल मंचों पर ही निर्भर करता था और इन मंचों पर मुख्यतः राजाओं, सम्राटों और रईसों का ही अधिकार होता था। अतः यह कला आम आदमी तक सही मापने में न के बराबर ही पहुँच पाती थी। रेडियो ने संगीत कला को आम समाज के लिए सुलभ बना दिया। मनोरंजन का यह सबसे सस्ता एवं श्रेष्ठ माध्यम माना गया। "भारत में रेडियो का आगमन और प्रचार प्रसार सन् 1936 ई॰ के लागभग हुआ, परन्तु संगीत की दृष्टि से रेडियों में प्रसारित क्रार्यक्रमों का पुनरूत्धान 1950 के बाद तत्कालीन सूचना तथा प्रसारण यन्त्री डा॰ बी.वी. केसकर द्वारा हुआ।"vi रेडियों की सेवाओं के कारण जनसाधारण में संगीत इतना सुलभ हो गया कि इसके प्रसारण द्वारा बड़े-बड़े कलाकारों का गायन वादन घर बैठे ही सुना जा सकता था।

\section{टेपरिकार्डर}

संगीत शिक्षण में टेपरिकार्डर की विशेष महत्त्ता है। यह एक ऐसा यन्त्र है जिसके माध्यम से प्रत्येक कार्यक्रम रिकार्ड किए जा सकते है तथा बार-बार सुन कर सीखे जा सकते हैं।इसकी 
सहायता से आकाशवाणी, दूरदर्शन या किसी भी संगीत सम्मेलन के कार्यक्रम को ज्यों का त्यो रिकार्ड किया जा सकता है तथा उस कार्यक्रम को बार-बार सुनने के लाभ उठाया जा सकता है शिक्षक द्वारा प्राप्त संगीत शिक्षण में यह उपकरण सहायक सिद्ध होता है। शिक्षक किसी भी संगीतज्ञ कार्यक्रम को रिकार्ड कर विद्यार्थियों को सुना सकता है। इससे विद्यार्थी अपने संगीत अभ्यासों की रिकार्ड कर सकते हैं और फिर शिक्षक के साथ अपनी त्रुटियों पर विचार-विमार्श कर सकते हैं। उन्हें अनुकरण एवं वारिन्तणिक कला में तुलना करने का अवसर मिलता है। नेत्रहीन विद्यार्थियों की सुविधा हेतु क्रियात्मक पक्ष के साथ शास्त्र पक्ष को भी इसमें रिकार्ड करके सहायक होता है।

\section{कॉम्पैक्ट डिस्क}

आधुनिक समय में कॉम्पैक्ट डिस्क का विशेष स्थान है। रिकॉर्ड प्लेयर की तुलना में यह तकनीक कहीं अधिक विकसित हुई है। इसकी सबसे बड़ी विशेषता यह है कि इसे अधिक समय तक सुरक्षित रखा जा सकता है। इसी प्रकार वाद्यों तथा कलाकारों की आवाज़ को इसमें अति सूक्ष्म एवं स्पष्टता से सुना जा सकता है।

\section{श्रव्य-दृश्य साधन}

दृश्य-श्रव्य सामग्री का तात्पर्य उन साधनों से है जिनमें श्रव्य दृश्य दोनों इन्द्रियों का प्रयोग होता है। अर्थात् जिनमें ज्ञान को श्रव्य तथा दृश्य दोनों इन्द्रियों द्वारा प्राप्त होता है। इनके अर्न्तगत ऐसे उपकरण आते हैं जिसके द्वारा प्रसारित क्रार्यक्रमों को देखा तथा सुना भी जा सकता है। ऐसे उपकरण बिजली की सहायता से चलते हैं। जिनमें टैलीविजन, सिनेमा, कम्पूयटर, कैसेट, सी. डी. इत्यादि आते हैं।

\section{टैलीविजन}

विद्यार्थियों के लिए टैलीविजन एक आकर्षण केन्द्र है। यह एक जीवंत साधन है अर्थात् किसी भी बात को सर्वथा विकार मुक्त रूप में उसी क्षण पर्दे पर प्रस्तुत करता है जब वह घटित होती है। संगीत-गोष्ठियाँ, संगीत सम्मेलन, सेमिनार, प्रश्नोत्तरी आदि कुछ टैलीविजन पर आधारित होने वाले कार्यक्रम है जिन्हें देखकर तथा सुन कर विद्यार्थी बिना परिश्रम के उनमें निहित संगीतात्मक सामग्री आत्मसात् कर लेते है। गायन-वादन-नृत्य तीनों की दुरदर्शन द्वारा व्यापक प्रसार हुआ है। सुगम, लोक व शास्त्रीय संगीत की व्यापता का श्रेय काफी हद तक दूरदर्शन को जाता है। दूरदर्शन के कार्यक्रम के अर्न्तगत देश के सुविख्यात कलाकारों का गायन, वादन व नृत्य प्रसारित किया जाता है। कार्यक्रम को आरम्भ करने से पूर्व कलाकार की शैलीगत विशेषताओं एवं उपलब्धियों पर प्रकाश डाला जाता है, जिससे दर्शक इन कलाकारों से भली-भांति परिचय प्राप्त करते है। 


\section{कम्पूयटर}

"अग्रेंज़ी शब्द शब्वचनजमतश से कम्पयूटर शब्द की उत्पत्ति हुई है, जिसका अर्थ गणना करना है, लेकिन आज इसका कार्य गणना करना ही नहीं, वरन सूचना देना तद्नुसार संगणना (च्तवबमेपदह) करना है।" vii संगीत शिक्षा एवं सांगीतिक अनुसंधान के क्षेत्र में कम्पूयटर की एक महत्वपूर्ण भूमिका है। इससे विभिन्न दिशाओं की संगीत सम्बन्धी प्रत्येक जानकारी प्राप्त होती है, संगीत सम्बन्धी विषयों पर शोध करने वाले छात्र संकलन सामग्री का विश्लेषण करने के लिए इसका प्रयोग करते हैं। 20 वी शताब्दी में कम्पूयटर का अभूतपूर्ण विकास हुआ। इससे संगीतक ध्वनि की आवृति, तारता आदि गुण नाप जा सकते है। कम्पूयटर में जटिल प्रोग्राम की सहायता से इनके इलावा संगीत की धुन एवं राग भी पहचाने जा सकते हैं और सूक्ष्म से सूक्ष्म त्रुटियाँ भी मालूम कर सकते हैं। इसकी सहायता से ध्वनि ही नहीं बल्कि संगीत की धुनों और रागों की रचना भी की जा सकती है।

\section{मंच प्रदर्शन}

"संगीत का काव्य कलाओं के प्रदर्शन के लिए बनाया गया वह स्थान श्रोताओं व रसिकों के स्थान से कुछ ऊंचा या नीचा एवं श्रोताओं के आकर्षण का केन्द्रबिन्दु हो, मंच कहलाता है।"viii संगीतकार की कला साधना का सबसे बड़ा एवं मुख्य उद्देश्य है मंच प्रदर्शन। मंच प्रदर्शन द्वारा विद्यार्थी प्रेरणा प्राप्त करता है। कलाकार के गुण एवं अवगुण देखता है। संगीतकार का मंच पर बैठना, उसके हाव-भाव, मुख आकृति, साज़ की सही पकड़, राग का आलाप, राग का स्वर विस्तार बंन्दिश पेशकारी, राग का स्वरूप, तबला वादक का कमाल, दोनों का आपसी मेल, विशेष बातों को देखना तथा सुन कर जो ज्ञान और आनन्द प्राप्त होता है वह किसी और माध्यम द्वारा नहीं हो सकता।

इस तरह दृश्य-श्रव्य वैज्ञानिक उपकरणों के प्रयोग से निश्चय ही संगीत शिक्षण बड़ा सरल, सुविधाजनक एवं रोचक हो जाता है। इन साधनों से विद्यार्थियों के मन पर इसका प्रभाव स्थायी होता है। एक अध्यापक का कर्तव्य विद्यार्थियों को संगीत शिक्षण द्वारा ज्ञान में वृद्धि करना है ताकि विद्यार्थी ज्यादा से ज्यादा अभ्यास कर अपने विषय में निपुण हो सके। विद्यार्थियों का अपने विषय में अग्रसर होना ही अध्यापक के लिए ईनाम है। इस लिए एक संगीत शिक्षक को विद्यार्थियों को आगे बढ़ने के लिए संभव कोशिश करनी चाहिए। 


\section{टिप्पणियाँ}

i M.S. Chhikara, Audio-Visual Education, Tondon Publication, Ludhiana, 1991, p.1.

ii ज्योति खन्ना, संगीत अध्यापन, पृ० 93.

iii एस. भटनागर, संगीत शिक्षण, पृ॰ 73, मोनिका प्रकाशन, शिमला, 1978.

iv .वही., पृ० 74,

v ज्योति खन्ना, संगीत अध्यापन, पृ॰ 91.

vi श्री खण्डे, हिन्दुस्तानी गायन की शिक्षा प्रणाली, पृ० 286.

vii शरदेन्दु किसलय, शिक्षण तकनीन, पृ० 230. डिस्कवरी पब्लिशिंग हाऊस, नई दिल्ली, 2006. viii राकेश बाला सक्सेना, मधुबाला सक्सेना, संगीत मधुबन, पृ॰ 204. 